DOI https://doi.org/10.30525/978-9934-588-81-5-1.45

\title{
INVESTIGATION OF SEPTIC CONDITIONS IN PATIENTS WITH BURN INJURY. METHOD OF FLUORESCENT SPECTROSCOPY
}

\author{
Savchyn V.S. \\ Candidate of Medical Sciences, \\ Head of Burn Department \\ Communal Clinical Hospital № 8 \\ Ostapiuk L. R. \\ Candidate of Medical Sciences, \\ Obstetrician-Gynecologist \\ Communal Non-profit Enterprise of the Lviv Regional Council \\ "Lviv Regional Public Health Centre"

\section{Tuziuk N. V.} \\ Postgraduate \\ Ivan Horbachevsky Ternopil National Medical University \\ of the Ministry of Health of Ukraine \\ Ternopil, Ukraine, \\ Doctor Surgeon-Combustiologist \\ Communal Clinical Hospital № 8 \\ Lviv, Ukraine
}

Burns occupy one of the leading places in the structure of injuries. Despite the introduction of modern advances in resuscitation and intensive care into medical praxis, the mortality of patients with widespread burns remains quite high.

According to the $\mathrm{WHO}$, the problem of burn injuries is currently one of the most global in the field of health care. There are up to 180.000 burn deaths worldwide each year, most of them in low- and middle-income countries. The infant mortality rate from burns in low-income countries is more than 7 times higher than in highly developed countries. Burns are one of the leading causes of morbidity, leading to long-term hospitalization, distortion and disability and are often accompanied by stigma and rejection. This is especially true for burns of the head and neck $[1,2]$. According to various surveys, these injuries occur from 55\% to $70 \%$ [1-4]. 
Despite current advances in prevention and treatment, sepsis remains the leading cause of death from severe burn injuries. It should be stressed that in these patients, early surgical closure of wounds significantly reduces the likelihood of systemic inflammatory response syndrome, hypermetabolism-hypercatabolism and possible manifestations of organ failure. Thus, a number of studies were aimed at finding and developing an appropriate algorithm for the diagnostics of burn sepsis [3,4]. Risk factors for sepsis include patients with various disorders of skin integrity: wounds, burns and skin infections. It is well known that the skin is a natural barrier of the body that performs the function of thermoregulation. After a burn injury, this barrier is broken and colonized by bacteria and fungi that increases the risk of developing early sepsis. In addition, the integrity of the intestinal barrier is disrupted, leading to the translocation of bacteria and endotoxins. Burn trauma with the area of more than $15 \%$ of the body surface leads to the development of hypovolemic shock and, as a consequence, to the systemic release of cytokines with the development of a generalized inflammatory reaction. The systemic inflammatory response with a large burn area includes systemic inflammation, endocrine stress response, and hypermetabolism, leading to circulatory hyperdynamics, increased oxygen demand, carbon dioxide production, glycolysis, proteolysis, lipolysis, and cyclic substrate processing. Pathophysiological changes in burn injury differ in different phases of burn disease (the early phase is up to 48 hours, and the late phase is 48 hours after burn).

The key to effective treatment of sepsis is its reliable and early diagnostics. Due to the latest advances in medicine at the turn of the late 20th and early 21 st century, much research has been devoted to these issues. Of course, they are all interesting and promising. Nevertheless, the issue of early diagnosis of sepsis remained relevant and unresolved.

Successful diagnostics should be based on the deep understanding of the pathogenesis of the disease at the molecular level. In recent years, physical research methods have become increasingly popular, which are characterized by high sensitivity and accuracy. The method of fluorescence spectroscopy (MFS) is the most universal method in biological spectroscopy. In $[5,6]$, this method was first used for studying purulent-inflammatory diseases, including sepsis. It is based on the profound understanding of the processes that occur in the body during the purulent-septic condition. Within the framework of MFS, the excitation of blood serum (BS) is performed by light with the wavelength of $280 \mathrm{~nm}$ which corresponds to the region of the excitation of human serum albumin. However, in the presence of endogenous intoxication in the body there are conformational changes of albumin molecules. 
Its binding centres are blocked by the products of bacterial metabolism. This changes the spectral-fluorescent characteristics of BS. In particular, there is a marked decrease in the intensity of fluorescence $\left(\mathrm{I}_{\mathrm{F}}\right)$, and in severe forms of the disease there is also a notable long-wave shift of the fluorescence band of BS $\left(\lambda_{\max }\right)$.

It is fundamentally important that these changes are registered 24-48 hours before the appearance of obvious clinical and laboratory signs of the source of infection. As a result, this approach is relevant in terms of prognostic assessment, monitoring and management of treating process, especially in sepsis.

The main aim of the study was to develop a method of diagnostics and treatment of patients with burn traumas, in which endogenous intoxication plays an important role [7].

The main group consisted of 12 patients, and the comparison group covered 10 patients with severe burn injuries. For the main group, the fluorescence spectra of BS were studied in the dynamics within the MFS, and the treatment algorithm was modified using the infusion of $10 \%$ albumin solution. For the comparison group, clinical and laboratory examination was performed and modified treatment tactics was also used.

Spectral-fluorescent characteristics for patient 1 (burn surface area 28\%) and patient with sepsis are presented in Fig. 1 and in Table 1.

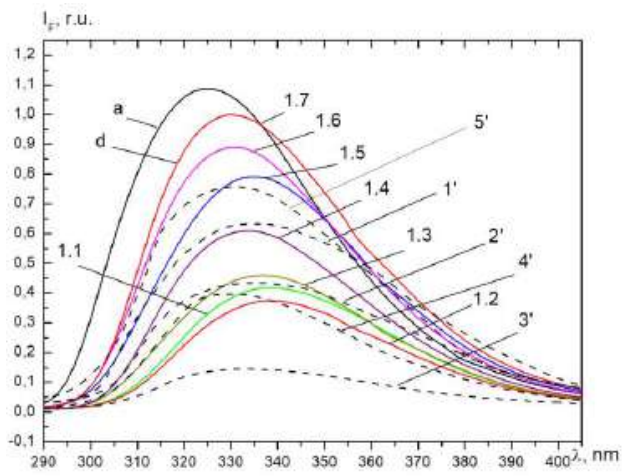

Fig. 1. FS of BS of patient 1 with burn injury, who was hospitalized at Communal Clinical Hospital No 8 in 2017 in dynamics during treatment (1.1 - 9.02., 1.2 - 14.02., 1.3 - 22.02., 1.4 -27.02.,1.5 - 03.03., 1.6 - 10.03., 1.7 - 31.03), and a patient with sepsis, who was treated in 2002 in Ambulance hospital $\left(1^{\prime}\right.$ - 03.06., $2^{\prime}-05.06 ; 3^{\prime}$ - 06.06; $\left.4^{\prime}-07.06,5^{\prime}-10.06\right)$ and $20 \%$ albumin solution $(a), \lambda_{\text {ex }}=280 \mathrm{~nm}$. 
Table 1

Spectral-fluorescence parameters (fluorescence intensity $\left(\mathbf{I}_{\mathbf{F}}\right)$ and the position of the maximum fluorescence band $\left(\lambda_{\max }\right)$ ) of blood serum of patient 1

\begin{tabular}{|c|c|c|c|c|c|c|c|c|c|c|c|c|c|c|}
\hline № & $\mathrm{a}$ & $\mathrm{d}$ & $1^{\prime}$ & $2^{\prime}$ & $3^{\prime}$ & $4^{\prime}$ & $5^{\prime}$ & 1.1 & 1.2 & 1.3 & 1.4 & 1.5 & 1.6 & 1.7 \\
\hline $\begin{array}{c}\lambda_{\max } \\
\mathrm{nm}\end{array}$ & 330 & 333 & 335 & 335 & 334 & 331.6 & 331 & 335 & 339 & 337 & 334 & 335 & 331 & 332 \\
\hline $\begin{array}{c}\mathrm{I}_{\mathrm{F}} \\
\mathrm{r.a}\end{array}$ & 1 & 1 & 0.63 & 0.43 & 0.14 & 0.4 & 0.76 & 0.41 & 0.37 & 0.46 & 0.61 & 0.79 & 0.89 & 0.95 \\
\hline
\end{tabular}

For patient 1, there was a significant amount of infusion therapy (more than 2 litres daily), but his $\mathrm{I}_{\mathrm{F}}$ of $\mathrm{BS}$ was low (for $1.1 \mathrm{I}_{\mathrm{F}}=0.41$ r.u., and for $1.2 \mathrm{I}_{\mathrm{F}}=0.37$ r.u.). His condition at the time of admission to the hospital was serious. During the further treatment, including effective antibiotic therapy, as well as the infusion of $10 \%$ solution of donor albumin $(06.02,10.02,15.02,18.02) \mathrm{I}_{\mathrm{F}}$ began to increase from 0.46 r.u. (curve 1.3) up to 0.95 r.u. (curve 1.7). After that, the patient was discharged from the hospital in satisfactory condition.

Similarly, the same change in the spectral-fluorescent characteristics of BS occurred in the patient with sepsis (Fig. 1). The difference between the results of these patients is in the values between the $\mathrm{I}_{\mathrm{F}}$ in the septic state. In general, due to the infusion of $10 \%$ albumin solution in the treatment of patient 1, the fluorescence intensities for him are greater than for patients without albumin infusions. Thus, we installed, that for patient 1 septic condition was realized. The condition of the other 10 patients in the main group corresponds to the condition of pre-septic pathology for surgical patients [8]. In some cases, this condition is close to septic.

It has been found that the structure of the fluorescence spectra of $\mathrm{BS}, \mathrm{I}_{\mathrm{F}}$ and $\lambda_{\max }$ are effective markers of the severity of burn injury and its prediction, which allow to assess the risk of critical purulent-septic complications and sepsis qualitatively and quickly.

Thus, we successfully used MFS to diagnose endogenous intoxication for patients of the main group and monitor their condition. The behaviour of spectral-fluorescent characteristics of the BS of patients with different severity was thoroughly studied. Standard treatment regimens have been improved. Of particular note is the proposal for the use of infusion of donor albumin solutions in case of the deterioration of patients' condition. This ensured a successful treatment process and proper control. 


\section{References:}

1. Savchyn, V. S. Features of reparative processes in patients with deep burns of the head and neck. Archive of clinical and experimental medicine. Volume 23, № 2, 2014. - P. 149-152.

2. Savchyn, V. S. Active surgical tactics in the treatment of head and neck burns. Hospital surgery №1, 2014. - p. 72-74 ISSN 1681-2778.

3. Kozynets, G.P., Sorokina, O.Yu. Slesarenko, S.V., Philip., Zh.V. Modern definition of sepsis and septic shock in patients with deep burns. Surgery of Ukraine.- 2017. - № 1. - P. 109-117. ISSN 1818-5398.

4. Sorokina, O.Yu., Koval, M.G. Screening and diagnosis of sepsis in severe burns. Emergency medicine. Volume 16, №1, 2020. - P. 16-22. ISSN 2307-1230.

5. Herych, I.D., Bulavenko, O.V. and Ostapiuk, L.R. (2014) SpectralFluorescent Properties of Serum as a Reliable Marker for Early Diagnosis of Sepsis. Journal Gynecology and Obstetrics, 2, 71-74.

6. Ostapiuk, L. (2019) Diagnostic and Therapeutic Model of Sepsis and Purulent-Inflammatory Diseases. International Journal of Clinical Medicine, 10, p. 577-595. https://doi.org/10.4236/ijcm.2019.1011047.

7. Zaporozhan, S., Savchyn, V., Ostapiuk, L., Voloshinovskii, A., Tuziuk, N. and Malyi, T. (2020) The New Approach to the Diagnostics and Treatment of Endogenous Intoxication in Patients with Burn Injury. International Journal of Clinical Medicine, 11, 375-388. https://doi.org/ 10.4236/ijcm.2020.116033.

8. Herych, I., Bulavenko, O., Ostapiuk, L., Voloshynovskyi, A. and Miahkota, S. (2015) Fluorescent Spectroscopy: ability to use in medical practice. Liha-Pres: 366. 